\title{
Association between Sleep Disturbances and Cardiovascular Diseases: Results from NHANES
}

\author{
Thierry P. Ayafor ${ }^{1 *}$, Jinkal Modi¹, Dayana A. Venkata1, Bolanle Bolaji1, Nicole C. Wright1,2 \\ ${ }^{1}$ Department of Epidemiology, School of Public Health, University of Alabama at Birmingham, Birmingham, AL, USA \\ ${ }^{2}$ Division of Clinical Immunology and Rheumatology, School of Medicine, University of Alabama at Birmingham, Birmingham, \\ AL, USA \\ Email:*pechapt@yahoo.fr,pechapt@yahoo.fr,jinkalm@uab.edu,dayana1@uab.edu,bbolaji@uab.edu,ncwright@uab.edu
}

How to cite this paper: Ayafor, T.P., Modi, J., Venkata, D.A., Bolaji, B. and Wright, N.C. (2020) Association between Sleep Disturbances and Cardiovascular Diseases: Results from NHANES. Journal of Biosciences and Medicines, 8, 1-11.

https://doi.org/10.4236/jbm.2020.84001

Received: February 15, 2020

Accepted: March 24, 2020

Published: March 27, 2020

Copyright $\odot 2020$ by author(s) and Scientific Research Publishing Inc. This work is licensed under the Creative Commons Attribution International License (CC BY 4.0).

http://creativecommons.org/licenses/by/4.0/

(c) (i) Open Access

\begin{abstract}
Objectives: The relationship between sleep disturbances and cardiovascular disease (CVD) is not well established. This study assesses the association between sleep disturbances and CVD, and the effect of sleep duration on the relationship between sleep disturbances and CVD among adults in the United States (US). Design: Cross-sectional analysis. Setting: NHANES (National Health and Nutrition Examination Survey). Participants: A total of 5660 adults were included from the 2015-2016 cycle of the NHANES survey. Measurements: The main outcome was the presence of any CVD and included self-reported angina, congestive heart failure, coronary heart disease, hypertension and myocardial infarction. Associations between sleep disturbances and sleep duration with CVD were analyzed using logistic regression. Stratified models by sleep duration were used to assess effect modification. Results: We included 5660 participants (52.2\% males), 32.7\% of the participants reported having a disturbed sleep and $38 \%$ reported a CVD. Compared to those who did not report any sleep disturbances, those with sleep disturbance had $85 \%$ higher odds of CVD (OR 1.85, 95\% CI 1.43 - 2.39). Similarly, there were $40 \%$ higher odds of CVD (OR 1.40, 95\% CI 1.01 - 1.95) among those with shorter sleep duration compared with those that slept for 6 to 9 hours. However, there was no evidence of effect modification by sleep duration. Conclusions: Our findings show that sleep disturbance is associated with higher odds of CVD. Clinicians and other healthcare providers need to consider the consequence of sleep disturbances and implement strategies in the treatment of patients with or at high risk of CVD.
\end{abstract}

\section{Keywords}

Sleep Disturbance, Cardiovascular Disease, Sleep Duration, NHANES 


\section{Introduction}

Sleep is a crucial function of health, coordinated by the brain; consisting of interplays between rapid eye movement and non-rapid eye movement sleep (NREM) [1]. For decades, sleep has been regarded as an essential lifestyle behavior that can impact cardiovascular disease (CVD) and mortality [2]. The American Academy of Sleep Medicine (AASM) and the Center for Disease Control and Prevention (CDC) recommend adults (18 - 64 years) should have at least 7 or more hours of sleep per night on a regular basis to optimize health [3] [4]. Over $10 \%$ of the United States (US) adults suffer from sleep disturbances with insomnia, sleep apnea and restless legs syndrome cited as the most common causes [5]. Symptoms are relatively more common among women, and increase with age [6]. Sleep disorders have been associated with a reduced quality of life and a heavy financial loss in damages and lost productivity [6].

Cardiovascular diseases (CVDs) are a leading cause of death in the US and globally [7]. Moreover, there is growing evidence that sleep disorders may adversely impact cardiovascular health [8]. In 2016, the American Heart Association closely examined evidence relating to sleep disorders and CVD and asked health organizations to come up with sleep recommendations regarding a number of sleep disorders [9]. Several epidemiological studies have reported an increased risk of CVDs or death in individuals with long sleep duration [10] [11], whereas other studies found contradictory results [12] [13] [14]. There is growing evidence of adverse effects of obstructive sleep apnea (OSA) on neurocognition and the cardiovascular system, with $4.7 \%$ of coronary heart disease being attributed to sleep disturbances [15].

Owing to the adverse effects of sleep-related disturbances, sleep disturbance is rapidly becoming a Public Health problem. Although several studies have examined the relationship between sleep duration and cardiovascular outcomes [16] [17] [18], there is little information from population-based studies showing association between the presence of sleep disturbance and all-cause cardiovascular events. To fill the knowledge gap, our study examined the association between self-reported sleep disturbances, as well as sleep duration and CVD. We also assessed the effect of sleep duration on the association between sleep disturbances and CVD. The National Health and Nutritional Examination Survey (NHANES) includes demographic, dietary, health related, and socioeconomic questions, making it a great data system for a population-based assessment on the association between sleep disturbances and CVD.

\section{Methods}

We queried the NHANES data and conducted a cross-sectional study of adults who participated in the 2015-2016 cycle. NHANES is a program of the National Center for Health Statistics (NCHS), a part of Centers for Disease Control and Prevention (CDC) in charge of producing national vital and health statistics. The goal of NHANES is to produce national estimates on a variety of health informa- 
tion, by conducting interviews, medical examinations, and laboratory analysis on a sample of participants [19]. The survey is conducted among a nationally representative sample of non-institutionalized participants, approximately 5000 persons each year, distributed in the different counties nationwide. We included participants $\geq 21$ years old who took part in NHANES 2015-2016 cycle and responded to our questions [19]. Loss of participants was due to missing CVD data $(\mathrm{n}=3856)$, missing sleep disturbance data $(\mathrm{n}=301)$ and missing sleep duration data $(n=174)$. The NHANES is a publicly available database, thus non-exempt human subject research.

\subsection{Main Exposures and Outcome Variables}

Our main exposure variable was having a sleep disturbance. Sleep disturbances were assessed by self-report based on the question "Have you ever told a doctor or other health professional that you have trouble sleeping?" with responses of "yes" or "no". To evaluate sleep duration, participants were asked "How much sleep do you usually get at night on weekends or workdays?" Responses ranged from 1 - 12 hours of sleep. We analyzed this variable both as a continuous and a categorical variable. Based on prior research [20] [21], we classified sleep duration into 3 categories; $\leq 6$ hours, $>6$ to $<9$ hours (reference), $\geq 9$ hours.

Our primary outcome was CVD. Participants self-reported CVD events based on the question "Has a doctor or health professional ever told you that you had [a particular disease]?" We classified participants as having a CVD if they reported any of the following conditions: angina, congestive heart failure, coronary heart disease, hypertension and myocardial infarction. Those responding "no" to all the diseases were placed in the no CVD group.

\subsection{Covariates of Interest}

Our socio-demographic characteristics included age, sex (male or female), race/ethnicity (White, African American, and Other), and income $(<\$ 20,000$, $\geq \$ 20,000$ ). Health variables included body mass index (BMI), depression, stroke, and diabetes. BMI was obtained based on participants' weights and heights. It was calculated by dividing weight by height in meters squared. We analyzed BMI both as a continuous variable and a categorical variable grouped into 4 categories: underweight $\left(<18.5 \mathrm{~kg} / \mathrm{m}^{2}\right)$, normal $\left(18.6-24.9 \mathrm{~kg} / \mathrm{m}^{2}\right)$, overweight $(25$ $\left.29.9 \mathrm{~kg} / \mathrm{m}^{2}\right)$, and obese $\left(\geq 30 \mathrm{~kg} / \mathrm{m}^{2}\right)$. Depression was assessed by the question "how often have you been bothered by feeling down, depressed, or hopeless?" with responses of "not at all, several days, more than half the days and nearly every day". Those who responded "more than half the days" or "nearly every day" were classified as having depression. The self-report of stroke and diabetes were based on the question "Have you ever been diagnosed of [medical condition]?".

\subsection{Statistical Analysis}

We conducted a bivariate analysis to assess association between covariates and 
outcome of interest using chi-square for categorical variables and T-test for continuous variables. Confounders were selected based on literature and statistical significance $(\mathrm{p}<0.05)$. Covariates that were statistically significant were included in the final model.

To assess the association between sleep disturbances and CVD, we calculated both the adjusted and unadjusted differences between both variables. Those without sleep disturbances were considered our reference category. We constructed a multivariable logistic regression model with CVD as outcome variable adjusting for significant covariates. We assessed the association between sleep duration and CVD, using logistic regression models; unadjusted model and adjusted models were used. Respondents who reported $>6$ to $<9$ hours of sleep were considered the reference category. To construct our adjusted model, potential confounders such as age, income, race, BMI, diabetes, stroke were added. Using multivariate logistic regression (adjusting for covariates which include age, income, BMI, depression, diabetes, stroke) we assessed the effect of sleep duration on the association between sleep disturbances and CVD with sleep duration as our interaction variable. All models utilized survey procedures to account for the complex survey design, and all statistical analysis was performed using SAS version 9.4 (SAS Institute Inc., Cary, NC).

\section{Results}

Our study sample included a total of 5660 participants (Figure 1). The mean age of the sample was 50.4 years (standard deviation $(\mathrm{SD})=0.40$ ) with a slightly higher proportion of females compared to males (52.2\% vs $47.8 \%$ ). We identified CVD in 2148 (38\%) of participants, with hypertension being the most predominant condition $(71.7 \%)$. The demographic and baseline characteristics of study

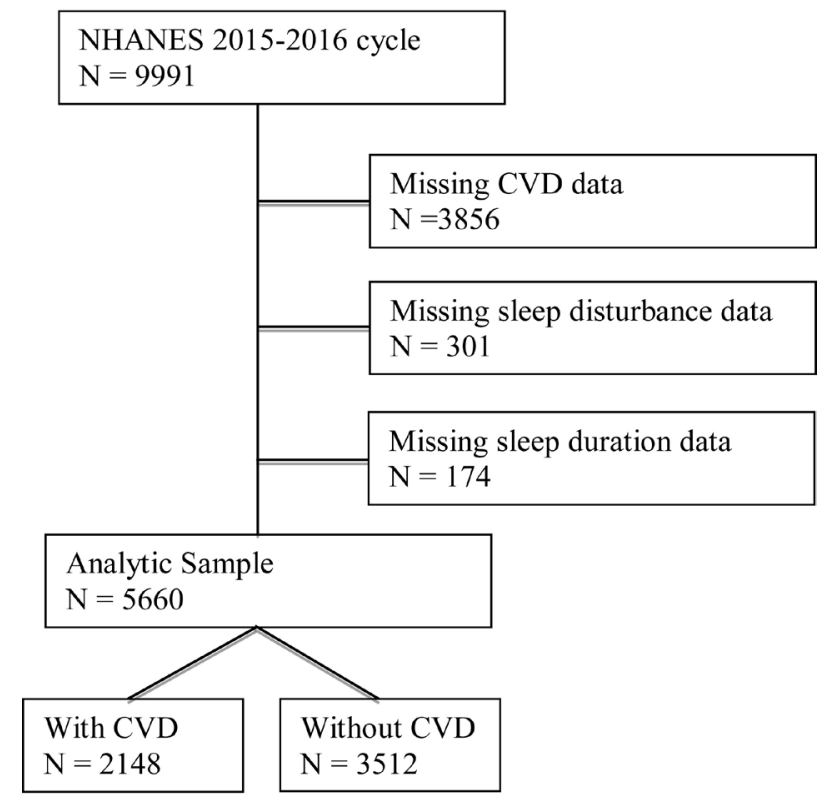

Figure 1. Flow diagram of study participants. 
participants by CVD status are reported in Table 1. Compared to participants in the non-CVD group, those in the CVD group were more likely to be African Americans $(25.8 \%$ vs. $18.2 \%)$, obese $(52.9 \%$ vs. $32.4 \%)$ and to report a low income status $(16.1 \%$ vs. $9.6 \%)(\mathrm{p}<0.001)$. Moreso, the CVD group were more likely to report having diabetes ( $26.8 \%$ vs. $5.9 \%)$, stroke ( $6.2 \%$ vs. $0.9 \%)$, and depression $(25.0 \%$ vs. $21.8 \%)$ ( $\mathrm{p}<0.001)$. Overall, the mean duration of sleep in our study was 7.7 hours $(\mathrm{SD}=0.04)$ with $25.8 \%$ of them reporting having trouble sleeping. Sleep disturbances were more prevalent in the participants with CVD (41.4\% vs. $23.9 \%, \mathrm{p}<0.001)$. Majority of our population $(61.1 \%)$ reported sleeping for $>6$ to $<9$ hours/day, $21.8 \%$ slept $\leq 6$ hours/day while $17.8 \%$ slept $\geq 9$ hours (data not shown).

Table 1. Demographics and baseline characteristics of participants.

\begin{tabular}{|c|c|c|c|}
\hline & $\operatorname{CVD}(\mathrm{N}=2148)$ & No CVD $(\mathrm{N}=3512)$ & $P$ value \\
\hline Age, mean $\pm S D$ & $58.3 \pm 0.4$ & $42.5 \pm 0.3$ & $<0.001$ \\
\hline \multicolumn{4}{|l|}{ Sex, n (\%) } \\
\hline Male & $1049(48.4)$ & $1658(47.7)$ & \multirow[t]{2}{*}{0.6667} \\
\hline Female & $1099(51.6)$ & $1854(52.3)$ & \\
\hline \multicolumn{4}{|l|}{ Race, n (\%) } \\
\hline African American & $554(25.8)$ & $638(18.2)$ & \multirow{3}{*}{$<0.001$} \\
\hline White & $731(34.0)$ & $1106(31.5)$ & \\
\hline Other & $863(40.1)$ & $1768(50.3)$ & \\
\hline \multicolumn{4}{|l|}{ Income, $\mathrm{n}(\%)$} \\
\hline$<20,000$ USD & $512(16.1)$ & $497(9.6)$ & \multirow[t]{2}{*}{$<0.001$} \\
\hline$>20,000$ USD & $1481(83.9)$ & $2754(90.4)$ & \\
\hline BMI $\left(\mathrm{kg} / \mathrm{m}^{2}\right)$, mean \pm SD & $31.6 \pm 0.2$ & $28.3 \pm 0.1$ & \\
\hline \multicolumn{4}{|l|}{ BMI, n (\%) } \\
\hline Underweight & $14(0.8)$ & $61(1.7)$ & \\
\hline Normal & $305(14.3)$ & $960(29.3)$ & \multirow{3}{*}{$<0.001$} \\
\hline Overweight & $666(32.0)$ & $1209(36.6)$ & \\
\hline Obesity & $1041(52.9)$ & $1096(32.4)$ & \\
\hline Hours of sleep, mean \pm SD & $7.60 \pm 0.05$ & $7.70 \pm 0.03$ & 0.5258 \\
\hline \multicolumn{4}{|l|}{ Sleep categories, $\mathbf{n}(\%)$} \\
\hline$\leq 6$ hours & $378(21.2)$ & $524(16.5)$ & \multirow{3}{*}{0.007} \\
\hline$>6$ to $<9 \mathrm{~h}$ & $903(62.7)$ & $1624(70.7)$ & \\
\hline$\geq 9$ hours & $312(16.1)$ & $391(12.8)$ & \\
\hline Sleep disturbances, $\mathrm{n}(\%)$ & $817(41.4)$ & $702(23.9)$ & $<0.001$ \\
\hline \multicolumn{4}{|l|}{ CVD conditions, $\mathbf{n}(\%)$} \\
\hline Angina & $205(7.1)$ & & \\
\hline Congestive heart failure & $235(8.3)$ & & \\
\hline Coronary heart disease & $130(4.6)$ & & \\
\hline Myocardial infarction & $235(8.3)$ & & \\
\hline Hypertension & $2035(71.7)$ & & \\
\hline \multicolumn{4}{|l|}{ Co-morbidities } \\
\hline Diabetes, n (\%) & $690(26.8)$ & $266(5.9)$ & $<0.001$ \\
\hline Stroke, n (\%) & $154(6.2)$ & $48(0.9)$ & $<0.001$ \\
\hline Depression, n (\%) & $509(25.0)$ & $665(21.8)$ & $<0.001$ \\
\hline
\end{tabular}

Abbreviations: $C V D$, Cardiovascular disease; $B M I$, Body Mass Index; $S D$, Standard Deviation. P value estimated using chi-square for categorical variable and t-tests for continuous variables. 
Table 2 represents the crude and unadjusted odds ratios for the association between sleep disturbances and CVD. In the crude model, those with sleep disturbances had 2.25-fold higher odds of CVD ( $\mathrm{OR}=2.25$, 95\% CI 1.85 - 2.72) than those without sleep disturbances; however, after controlling for age, BMI, race, income status, diabetes, stroke, and depression, the association attenuated. After adjustment, those with sleep disturbances had 85\% higher odds of reporting CVD than those without sleep disturbances (OR $=1.85(1.43-2.39), \mathrm{p}<$ $0.001)$.

Table 3 shows the crude and adjusted odds ratios for the association between sleep duration and cardiovascular diseases. Compared to participants with a normal sleep duration ( $>6-<9$ hours per night), those sleeping $\leq 6$ hours and $\geq 9$ hours per night had higher crude odds ratios for cardiovascular diseases of ( $\mathrm{OR}=$ $1.45,95 \% \mathrm{CI} 1.13-1.85)$ and $(\mathrm{OR}=1.42,95 \% \mathrm{CI} 1.14-1.78)$ respectively $(\mathrm{p}<$ 0.001). This relationship persisted after adjusting for age, BMI, race, income status, diabetes, stroke and depression; however, there was no evidence of an association between long sleep duration and CVD after adjustment $(\mathrm{OR}=1.19$ (0.90 - 1.60), $\mathrm{p}=0.28$ ).

Table 4 shows the crude and adjusted odd ratios for the effects of sleep duration on the association between sleep disturbances and CVD. There was no evidence of effect modification by sleep duration as shown by the overlapping confidence intervals even after adjusting for age, BMI, race, income status, diabetes, stroke and depression $(\mathrm{p}=0.15)$.

\section{Discussion}

\subsection{Main Findings of This Study}

In this study, we found that sleep disturbances were associated with CVD in a large representative US sample. After adjusting for our confounders, those with sleep disturbances had $85 \%$ higher odds of reporting CVD than those without sleep disturbances. Compared to participants with a normal sleep duration $(>6$

Table 2. Association between sleep disturbances and cardiovascular diseases.

\begin{tabular}{ccc}
\hline & Crude OR $(95 \% \mathrm{CI})$ & Adj. $_{\text {OR }}^{\dagger}(\mathbf{9 5 \%} \mathrm{CI})$ \\
\hline YES & $2.25(1.85-2.72)$ & $1.85(1.43-2.39)$ \\
NO & Ref & Ref \\
\hline
\end{tabular}

Abbreviation: Ref, reference group. ${ }^{\dagger}$ Adjusted by age, BMI, race, income status, diabetes, stroke, depression.

Table 3. Association between sleep duration and cardiovascular diseases.

\begin{tabular}{ccc}
\hline & Crude OR (95\% CI) & Adjusted OR $^{\dagger}(95 \% \mathrm{CI})$ \\
\hline$\leq 6$ hours & $1.45(1.13-1.85)$ & $1.40(1.01-1.95)$ \\
$>6$ to $<9 \mathrm{~h}$ & Ref. & Ref. \\
$\geq 9$ hours & $1.42(1.14-1.78)$ & $1.19(0.90-1.60)$ \\
\hline
\end{tabular}

Abbreviation: Ref, reference group. ${ }^{\dagger}$ Adjusted by age, BMI, race, income status, diabetes, stroke, depression. 
Table 4. Effect of sleep duration on the association between sleep disturbances and cardiovascular diseases.

\begin{tabular}{cccc}
\hline Sleep duration & Sleep disturbances & Crude OR $(95 \% \mathrm{CI})$ & Adj. $^{\mathrm{OR}^{\dagger}(95 \% \mathrm{CI})}$ \\
\hline$\leq 6 \mathrm{~h}$ & & $1.90(1.32-2.62)$ & $1.64(0.98-2.51)$ \\
$>6$ to $<9 \mathrm{~h}$ & YES & Ref & Ref \\
$\geq 9 \mathrm{~h}$ & & $1.83(1.21-2.74)$ & $1.54(0.55-2.8)$ \\
$\leq 6 \mathrm{~h}$ & & $1.16(0.82-1.51)$ & $1.21(0.87-1.78)$ \\
$>6$ to $<9 \mathrm{~h}$ & NO & Ref & $\begin{array}{c}\text { Ref } \\
\geq 9 \mathrm{~h}\end{array}$ \\
& & $1.22(0.87-1.70)$ & $1.07(0.72-1.58)$ \\
\hline
\end{tabular}

Abbreviation: Ref, reference group. ${ }^{\dagger}$ Adjusted by age, BMI, race, income status, diabetes, stroke, depression.

and $<9$ hours per night), those sleeping $\leq 6$ hours and $\geq 9$ hours per night had slightly higher crude odds ratios for cardiovascular diseases. This relationship persisted even after adjusting for age, BMI, race, income status, diabetes, stroke and depression; however, there was no evidence of an association between long sleep duration and CVD after adjustment. These findings suggest that short sleep durations are associated with cardiovascular diseases. The present study also found no evidence of effect modification by sleep duration as shown by the overlapping confidence intervals even after adjusting for our confounders.

\subsection{Comparing Our Findings to the Literature}

Our findings are similar to those who have evaluated the relationship between sleep disturbances and CVD. There's a paucity of literature regarding association between disturbed sleep and CVD. Few studies have found increasing evidence of association of sleep apnea and CVD [22]. Although we had a general sleep disturbance variable, these associations are similar to what we found in our study. Also, studies of insomnia have found a positive association with coronary heart disease [23]. Coronary heart disease was one of the conditions included in our overall CVD outcome. Likewise, there is growing evidence between the relationship between sleep disruption and subsequent increase in blood pressure [23].

Studies specifically evaluating sleep duration have found mixed results. Covassin et al. found a U-shaped relationship between self-reported sleep length and hypertension in a large $(\mathrm{N}=71,455)$ national representative sample $(\mathrm{Na}-$ tional Health Interview Survey, NHIS) with both ends of the tail exhibiting larger age-standardized prevalence of hypertension ( $<6$ hours/night: $32.4 \%$; $\geq 10$ hours/night: $32.5 \%$ ) compared to the referent category (8 hours/night, $23.2 \%$ ) [24]. Likewise, in a Japanese based study, they found that men who sleep $<6$ hours a day have a greater risk of developing cardiovascular events than those sleeping 7 to 7.9 hours [25]. A dysregulation in endocrine and metabolic functions have been speculated to account for the association between short sleep duration and CVD. A lack of sleep results in increased sympathetic activity, increased blood pressure, impaired glucose tolerance all of which augment the risk for atherosclerosis [26]. Sleep deprivation studies in rats have shown similar en- 
docrine and metabolic dysregulations [27].

However, in the pooled analysis of a systematic review and meta-analysis of prospective studies evaluating sleep duration on cardiovascular found that short sleep duration was not significantly associated with the greater risk of developing CVD [1.03 (0.93 - 1.15); $\mathrm{p}=0.52]$ while the long duration of sleep was strongly associated with the CVD [1.41 (1.19 - 1.68); p < 0.0001) [16]. In our study, we found that compared to participants with a normal sleep duration ( $>6$ and $<9$ hours per night), those with short sleep duration ( $\leq 6$ hours) did have a significant $40 \%$ increased odds for CVD, whereas, there was no evidence of an association with CVD in those with long sleep duration ( $\geq 9$ hours). The difference in these results is unclear. Future research is required to better understand the association between long sleep duration and CVD.

\subsection{What This Study Adds}

We found that sleep disturbances even when adjusted for age, BMI, race, income status, diabetes, stroke, and depression, was independently associated with cardiovascular diseases. This is the first study to investigate the effect modification with adjustment of known confounders. Our findings although includes observational study of one cycle, provides the basis needed to further explore the association between sleep disturbances and cardiovascular disease and how the trend has changed from then to now.

\subsection{Limitations and Strengths}

Our study has some strengths and limitations. NHANES is a nationally representative sample with a large sample size with both male and female included in our study. We modeled our outcome variable to include any cardiovascular disease. We adjusted for covariates in our study which include age, income, BMI, depression, diabetes, and stroke. Limitations of our study include secondary analysis of self-report may lead to misclassification of outcome and information bias. Our study is cross-sectional; causality and incidence rates cannot be determined. Also, the temporal relationship between sleep disturbance and cardiovascular diseases is questionable based on cross-sectional design. Residual confounding remains as we were unable to adjust for all factors potentially confounding the sleep disturbance and CVD relationship. To eliminate the possibility of information bias from self-report, future studies should employ a more objective approach to measure sleep quality, such as polysomnography and actigraphy, and objective medical data for covariates of interest. Future research should be conducted on obtaining medical records to confirm CVD diagnosis. Retrospective analysis may be conducted on association between trends of sleep duration and CVD.

\section{Conclusion}

We found that sleep disturbances are associated with higher odds of cardiovas- 
cular diseases. Likewise, shorter sleep durations were associated with CVD. However, we did not find any evidence of interaction between sleep duration and sleep disturbance. This study shows that sleep duration and sleep disturbances could be important modifiable risk factors for CVD. Sleep disturbances and CVD events incur tremendous economic costs relating to the loss of quality of life. Clinicians and other health professionals need to heavily consider the bifacial consequence of sleep disturbances and implement strategies in the treatment of patients with or a high risk of cardiovascular diseases.

\section{Conflicts of Interest}

The authors declare no conflicts of interest regarding the publication of this paper.

\section{Financial/Nonfinancial Disclosures}

None declared.

\section{Funding}

This project was not supported by any funding source.

\section{References}

[1] Reyfman, P.A., et al. (2018) Single-Cell Transcriptomic Analysis of Human Lung Reveals Complex Multicellular Changes During Pulmonary Fibrosis.

[2] Knutson, K.L. and Van Cauter, E. (2008) Associations between Sleep Loss and Increased Risk of Obesity and Diabetes. Annals of the New York Academy of Sciences, 1129, 287-304. https://doi.org/10.1196/annals.1417.033

[3] Watson, N.F., et al. (2015) Joint Consensus Statement of the American Academy of Sleep Medicine and Sleep Research Society on the Recommended Amount of Sleep for a Healthy Adult: Methodology and Discussion. Sleep, 38, 1161-1183. https://doi.org/10.5665/sleep.4886

[4] Centers for Disease Control and Prevention (2019) Are You Getting Enough Sleep? https://www.cdc.gov/features/sleep/index.html

[5] Ram, S., et al. (2010) Prevalence and Impact of Sleep Disorders and Sleep Habits in the United States. Sleep and Breathing, 14, 63-70. https://doi.org/10.1007/s11325-009-0281-3

[6] Colten, H.R. and Altevogt, B.M. (2006) Institute of Medicine Committee on Sleep, M. and Research, The National Academies Collection: Reports Funded by National Institutes of Health, in Sleep Disorders and Sleep Deprivation: An Unmet Public Health Problem. National Academies Press (US) National Academy of Sciences, Washington DC.

[7] Mc Namara, K., Alzubaidi, H. and Jackson, J.K. (2019) Cardiovascular Disease as a Leading Cause of Death: How Are Pharmacists Getting Involved? Integrated Pharmacy Research \& Practice, 8, 1-11. https://doi.org/10.2147/IPRP.S133088

[8] Wolk, R., et al. (2005) Sleep and Cardiovascular Disease. Current Problems in Cardiology, 30, 625-662. https://doi.org/10.1016/j.cpcardiol.2005.07.002

[9] St-Onge, M.P., et al. (2016) Sleep Duration and Quality: Impact on Lifestyle Behaviors and Cardiometabolic Health: A Scientific Statement from the American Heart 
Association. Circulation, 134, e367-e386. https://doi.org/10.1161/CIR.0000000000000444

[10] Ikehara, S., et al. (2009) Association of Sleep Duration with Mortality from Cardiovascular Disease and Other Causes for Japanese Men and Women: The JACC Study. Sleep, 32, 295-301. https://doi.org/10.1093/sleep/32.3.295

[11] Kim, Y., et al. (2013) Insufficient and Excessive Amounts of Sleep Increase the Risk of Premature Death from Cardiovascular and Other Diseases: The Multiethnic Cohort Study. Preventive Medicine, 57, 377-385. https://doi.org/10.1016/j.ypmed.2013.06.017

[12] Stone, K.L., et al. (2009) Self-Reported Sleep and Nap Habits and Risk of Mortality in a Large Cohort of Older Women. Journal of the American Geriatrics Society, 57, 604-611. https://doi.org/10.1111/j.1532-5415.2008.02171.x

[13] Hall, M.H., et al. (2015) Association between Sleep Duration and Mortality Is Mediated by Markers of Inflammation and Health in Older Adults: The Health, Aging and Body Composition Study. Sleep, 38, 189-195. https://doi.org/10.5665/sleep.4394

[14] Jung, K.I., et al. (2013) Gender Differences in Nighttime Sleep and Daytime Napping as Predictors of Mortality in Older Adults: The Rancho Bernardo Study. Sleep Medicine, 14, 12-19. https://doi.org/10.1016/j.sleep.2012.06.004

[15] Malhotra, A. and Loscalzo, J. (2009) Sleep and Cardiovascular Disease: An Overview. Progress in Cardiovascular Diseases, 51, 279-284. https://doi.org/10.1016/j.pcad.2008.10.004

[16] Cappuccio, F.P., et al. (2011) Sleep Duration Predicts Cardiovascular Outcomes: A Systematic Review and Meta-Analysis of Prospective Studies. European Heart Journal, 32, 1484-1492. https://doi.org/10.1093/eurheartj/ehr007

[17] Sabanayagam, C. and Shankar, A. (2010) Sleep Duration and Cardiovascular Disease: Results from the National Health Interview Survey. Sleep, 33, 1037-1042. https://doi.org/10.1093/sleep/33.8.1037

[18] Kwok, C.S., et al. (2018) Self-Reported Sleep Duration and Quality and Cardiovascular Disease and Mortality: A Dose-Response Meta-Analysis. Journal of the American Heart Association, 7, e008552. https://doi.org/10.1161/JAHA.118.008552

[19] Centers for Disease Control and Prevention (2015) National Health and Nutrition Examination Survey.

https://wwwn.cdc.gov/nchs/nhanes/continuousnhanes/overview.aspx?BeginYear=2 $\underline{015}$

[20] Cepeda, M.S., et al. (2016) Clinical Relevance of Sleep Duration: Results from a Cross-Sectional Analysis Using NHANES. Journal of Clinical Sleep Medicine, 12, 813-819. https://doi.org/10.5664/jcsm.5876

[21] Xiao, Q., et al. (2013) A Large Prospective Investigation of Sleep Duration, Weight Change, and Obesity in the NIH-AARP Diet and Health Study Cohort. American Journal of Epidemiology, 178, 1600-1610. https://doi.org/10.1093/aje/kwt180

[22] Jean-Louis, G., et al. (2008) Obstructive Sleep Apnea and Cardiovascular Disease: Role of the Metabolic Syndrome and Its Components. Journal of Clinical Sleep Medicine: JCSM: Official Publication of the American Academy of Sleep Medicine, 4, 261-272. https://doi.org/10.5664/jcsm.27191

[23] Javaheri, S. and Redline, S. (2017) Insomnia and Risk of Cardiovascular Disease. Chest, 152, 435-444. https://doi.org/10.1016/j.chest.2017.01.026

[24] Covassin, N. and Singh, P. (2016) Sleep Duration and Cardiovascular Disease Risk: Epidemiologic and Experimental Evidence. Sleep Medicine Clinics, 11, 81-89. 
https://doi.org/10.1016/j.jsmc.2015.10.007

[25] Amagai, Y., et al. (2010) Sleep Duration and Incidence of Cardiovascular Events in a Japanese Population: The Jichi Medical School Cohort Study. Journal of Epidemiology, 20, 106-110. https://doi.org/10.2188/jea.JE20090053

[26] Spiegel, K., Leproult, R. and Van Cauter, E. (1999) Impact of Sleep Debt on Metabolic and Endocrine Function. The Lancet, 354, 1435-1439. https://doi.org/10.1016/S0140-6736(99)01376-8

[27] Rechtschaffen, A., et al. (2002) Sleep Deprivation in the Rat: X. Integration and Discussion of the Findings. 1989. Sleep, 25, 68-87.

https://doi.org/10.1093/sleep/25.1.68 